\title{
IXe Conférence internationale du Radiocarbone et correction des
} dates $14 \mathrm{C}$

Georgette Delibrias, Jacques Évin, J. Thommeret, Y. Thommeret

\section{Citer ce document / Cite this document :}

Delibrias Georgette, Évin Jacques, Thommeret J., Thommeret Y. IXe Conférence internationale du Radiocarbone et correction des dates 14 C. In: Bulletin de la Société préhistorique française. Comptes rendus des séances mensuelles, tome 73 , ${ }^{\circ} 9$, 1976. pp. 268-269;

doi : https://doi.org/10.3406/bspf.1976.10289

https://www.persee.fr/doc/bspf_0249-7638_1976_num_73_9_10289

Fichier pdf généré le 08/09/2020 


\title{
IX Conférence internationale
}

\section{du Radiocarbone et correction des dates $14 \mathrm{C}$}

\author{
par G. Delibrias (1), J. Evis (2), J. et I. 'Thommereit (3)
}

Au cours de la $\mathrm{IX}^{\circ}$ Conférence internationale du Radiocarbone qui s'est tenue en juin 1976 aux Etats-Unis, il a été décidé que «l'usage de $\mathrm{AD}$ et $\mathrm{BC}$ pour annoter les nombres obtenus par soustraction de 1950 des dates conventionnelles ${ }^{14} \mathrm{C}$, serait abandonné immédiatement $》$.

Les laboratoires de datation par le radiocarbone de Gif, Lyon et Monaco vont devoir se conformer à cette décision internationale et ne plus donner la correspondance $\mathrm{AD} / \mathrm{BC}$ des dates ${ }^{14} \mathrm{C}$, mais exclusivement leur valeur $\mathrm{BP}$.

Les auteurs indiquent ici les raisons de cette décision et font aussi le point sur les corrections dendrochronologiques.

\section{Nécessité d'un langage commun.}

L'application stricte d'une seule forme de publication des résultats ${ }^{14} \mathrm{C}$ par tous : laboratoire de radiocarbone et auteurs archéologues ou géologues utilisant les dates, est la condition nécessaire pour qu'aucune confusion ne se produise dans l'interprétation des données de chronologie absolue.

L'unicité d'un mode d'écriture de cette chronologie semble de plus en plus indispensable par suite de l'augmentation considérable du nombre des datations. En outre, apparait depuis quelques temps un usage abusif de termes et de lettres tels que : " date $\mathrm{BC}$, date avant notre ère, date calibrée, date corrigée, date réelle », dont les définitions exactes ne sont ni précisées, ni mème, le plus souvent, bien interprétées par leurs utilisateurs. Il arrive même que, par confusion, des dates ne se référant pas aux mêmes normes soient comparés.

(1) Centre des Faibles radioactivités, cNRS-CEA, Gif-surYvette.

(2) I.aboratoire de Radiocarbone, Iyon.

(3) Laboratoire de radioactivité appliquée, C.s.M. Monaco.
I. - La correction des dates ${ }^{1+} \mathrm{C}$ all moyen de la dendrochronologie.

Il est maintenu bien établi qu'il peut exister un écart entre les âges ${ }^{14} \mathrm{C}$ ot les âges réels. Cet écart est variable suivant les époques et son ordre de grandeur est conmu jusqu'aux environs de l'âge radiocarbone $7500 \mathrm{BP}$, où il atteint environ 700 ans (les âges ${ }^{1+} \mathrm{C}$ étant trop faibles par rapport à ceux du calendrier solaire).

\section{Au-delà de 7500 BP.}

Des recherches sont en cours pour déterminer si au-delà de cette date l'écart est de plus en plus important ou si, ce qui semble être le cas, il aurait tendance à baisser ou même à s'inverser. Actuellement au-delà de cette date de $7500 \mathrm{BP}$ rien n'a encore été établi et il est absolument exclus de faire une quelconque correction. Toute date antérieure à $7500 \mathrm{BP}$ doit ètre considérée comme non corrigée et toute «date corrigée » ou « date calibrée » doit alors être considérée comme fausse.

\section{En deçà de $7500 B P$.}

L'écart possible entre une date ${ }^{14} \mathrm{C}$ et l'âge réel est, rappelons-le, dù à un écart entre la teneur en radiocarbone de l'atmosphère à l'époque de la formation de l'échantillon et la teneur en radiocarbone de l'atmosphère actuelle. Ceci veut dire que pour effectuer une correction valable il faut connaitre pour chaque année, ou au moins chaque groupe de 10 années, cette leneur primitive en ${ }^{14} \mathrm{C}$. L La mesure de la radioactivité d'anneaux d'arbres anciens très exactement datés par dendrochronologie permet de retrouver cette valeur en ${ }^{14} \mathrm{C}$ initiale. Il est important de savoir que cette mesure est délicate et doit ètre effectuée sur plusieurs échantillons d'âge voisin. La valeur obtenue reste entachée d'une certaine erreur. Les laboratoires spécialisés dans cette étude ne sont pas encore 
parvenus à un accord sur les facons de calculer ces valeurs, si bien qu'il existe actuellement plusieurs courbes de correction.

\section{La correction par les laboratoires.}

Pour cette raison, tant qu'un accord international n'aura pas eu lieu, nos laboratoires de radiocarbone se refuseront à publier des corrections dendrochronologiques et continueront comme par le passé à donner uniquement des âges ${ }^{14}(\mathrm{C}, \mathrm{BP}$.

Une des recommandations de la $I X^{*}$ Conférence du Radiocarbone, à toute personne voutlant, malgré les dangers de cette operation, effectuer une correction des âges ${ }^{1+} \mathrm{C}$, a été de bien préciser lors de chaque publication la courbe de correction choisie ainsi que l'origine des travaux invoqués. Il devrait alors être utilisé dans la rédaction la seule notation $\mathrm{AD}^{*} / \mathrm{BC}^{*}$.

Rappelons de plus que toute correction n'apporte pas une meilleure précision, car à l'erreur statistique de la date ${ }^{14} \mathrm{C}$ s'ajoute l'erreur sur la teneur en ${ }^{14} \mathrm{C}$ initiale mesurée lors de l'établissement de la courbe de correction et l'imprécision supplémentaire due aux petites fluctuations de cette courbe. Ces dernières ne sont pas du tout négligeables et on peut dire qu'à un âge ${ }^{14} \mathrm{C}$ précis correspond toute une plage de dates réelles. Il faut savoir que la précision de l'âge corrigé sera inférieure à celle qui aurail été donnée pour l'âge ${ }^{14} \mathrm{C}$ mesuré. Parfois elle sera au minimum deux fois plus faible que celle donnée sur cet âge.

\section{II. - Date $B P$, date $B C$.}

La IX' Conférence internationale du radiocarbone a décidé que désormais les dates ${ }^{14} \mathrm{C}$ ne seraient plus publices en $\mathrm{BC} / \mathrm{AD}$, mais uniquement en BP (Before Present), (le «Present 》 étant toujours par convention l'année 1950). En effet jus(u'a maintenant les dates $\mathrm{BC} / \mathrm{AD}$ ) ćtaient obtenues par soustraction de 1950 (année 0 de référence ${ }^{4} \mathrm{C}$ ). Mais il est bien civident que l'on arriverait à une confusion si les dates corrigées étaient, elles aussi, exprimées en $\mathrm{BC} / \mathrm{AD}$ ) même accompagnées d'un astérisque ou si les dates ${ }^{14} \mathrm{C}$ étaient notées en lettres minuscules ad, bc.

Il faut que l'habitude d'exprimer les dates en $B P$, pour les dates ${ }^{14} \mathrm{C}$ non corrigées soit désormais acquise d'une facon définitive. Il est regrettable que de récentes publications de synthèse donnent encore uniquement les dates ${ }^{14} \mathrm{C}$ en $\mathrm{BC} / \mathrm{AD}$ ).

La nouvelle décision internationale semble tout à fait justifiéc car elle prépare l'avenir : lorsque les mesures de la teneur en ${ }^{14} \mathrm{C}$ de l'atmosphère du temps passé pourront être considérées comme achevées, lorsque sera connue avec une précision suffisante la correction à appliquer à chaque date et que le mode de correction sera internationalement adopté, alors les laboratoires pourront publier à nouveau en date $\mathrm{BC}^{*}$ et $\mathrm{AD}^{*}$ en conservant la notation $\mathrm{BP}^{\mathrm{P}}$ pour la plage de temps où la correction ne sera pas encore possible.

Nous devons ìtre conscients que si l'habitude de faire la distinction entre $\mathrm{BP}^{\prime}$ et $\mathrm{BC}^{\star}$ n'est pas prise dès maintenant, l'imbroglio deviendra total dans quelques années.

Actuellement les archéologues utilisent le plus fréquemment les dates en $\mathrm{BC} / \mathrm{AI}$ ). Ils justifient l'emploi de ces dates ainsi exprimées, par la nécessite de comparaisons avec des dates calendaires vraies et par leur habitude de parler en Millénaires avant le Christ. Ainsi se comprend leur tendance à utiliser les âges ${ }^{1+} \mathrm{C}$ exprimées en $\mathrm{BC} / \mathrm{AD}$, mais il n'est scientifiquement possible de le faire que pour des dates corrigées, sinon il existe un danger de confusion et un risque d'oubli d'une date utilisée sans correction. Par exemple : un âge ${ }^{1+} \mathrm{C}$ de $4500 \mathrm{BC} \pm 110$ correspond (toutes corrections appliquées) à un événement de la fin du $6^{\circ}$ Millénaire, il devrait être noté : «environ 5200 $\mathrm{BC}^{*}$ » ou mieux «5290$5100 \mathrm{BC}^{*}$ » (Ralph et al., 1976), ou simplement sous la forme non corrigée : $6450 \mathrm{BP} \pm 110$.

\section{Conclusion.}

Les résolutions de la $\mathrm{IX}^{\circ}$ Conférence du radiocarbone (1976) demandent que désormais, toute date ${ }^{14} \mathrm{C}$ tant qu'elle n'aura pas été corrigée, soit toujours ètre exprimée sous la forme BP. La notation $\mathrm{BC}^{*}$ ne sera employée pour désigner une date sur laquelle il aura été effectué une correction, pour autant que celle-ci n'ait pas déjà été accomplie par les laboratoires. Il faut que cette forme de notation unique s'étende à toutes les publications.

Nous ne saurions trop insister sur la nécessité, pour les utilisateurs de nos travaux de datation de se soumettre à la discipline qu'entraine désormais les résolutions de cette Conférence. Nous espérons que ces dernières seront malgré tout suivies par le plus grand nombre, car nous avons conscience que l'amélioration des techniques ou l'accumulation d'un plus grand nombre de résultats deviendraient inutiles si, en fin de compte, nous aboutissions à une confusion quasi totale qui finirait par discréditer les travaux des utilisateurs comme des techniciens, et ceci par la simple absence d'une normalisation librement consentie.

\section{BIBIIOGRAPHIE:}

Ralph K., Michafe H. N. \& Hax M. C. (1976). Tree rings and $1+\mathrm{C}_{1}$ scale : Colloque sur les datations absolues et analyses isotopiques en Préhistoire. méthodes et limites. Nice, $13 / 18$ sept. 1976, p. 126. 